\title{
La couleur des solutions d'iode à basse température
}

\author{
par \\ Jean Piccard et E. Herrmann.
}

(31. V. 12.)

Dans une récente communication ${ }^{1}$ ) sur la coloration brune produite par l'iode dans des solutions organiques contenant de l'oxygène, j'ai démontré que cette coloration pouvait être employée comme réactif pour la détermination qualitative de l'oxygène dans des liquides organiques. Cette réaction repose sur la formation d'un produit d'addition ${ }^{2}$ ) de l'iode à un élément coordinativement non saturé, dans ce cas à l'oxygène ${ }^{3}$ ). Des corps contenant de l'azote trivalent ou du carbone avec une valence de coordination disponible présentent un phénomène analogue et il est évident que l'on ne pourra se servir de l'iode pour révéler la présence de l'oxygène qu'en l'absence d'autres éléments coordinativement fortement non saturés.

A ce sujet, M. le prof. Constant Dutoit a bien voulu me communiquer l'observation suivante: les solutions violettes d'iode dans des hydrocarbures tels que la ligroïne virent au brun à basse température, si elles ne sont pas trop concentrées. Mon collègue, M. Dutoit a eu l'amabilité de m'autoriser à étendre mes recherches à ce domaine et je profite de cette occasion pour lui en présenter mes remerciments sincères.

J. $P$.

Si l'on dissout environ $1 \mathrm{mg}$. d'iode dans $10 \mathrm{~cm}^{3}$ de ligroïne, on obtient une solution violette qui vire au brun foncé lorsqu'on la refroidit à une température de $-15^{\circ}$ à $-20^{\circ}$.

La coloration brune n'est pas due à la formation d'un produit de polymérisation de l'iode comme nous l'avons cru d'abord. Elle doit résulter d'une addition de la molécule d'iode à un autre corps, puisque la concentration du corps brun cesse bientôt d'augmenter avec celle de l'iode. En effet, comme M. Dutoit l'avait déjà remarqué, le phénomène disparaît avec l'augmentation de la teneur en iode. Il découle également de cette constatation que le second composant doit se trouver en concentration très faible (de l'ordre de grandeur de $1 \mathrm{mg}$. par $10 \mathrm{~cm}^{3}$ ).

1) Helv. 5, 243 (1922).

$\left.{ }^{2}\right)$ Beckmann et Stock, Z. ph. Ch. 17, 107 (1895); Vaupel, J. pr. Ch. [2] 63, 318 (1901); Hantzsch et Denstorff, A. 349, 1 (1906).

3) On peut démontrer le fait qu'il s'agit vraiment d'une réaction chimique par l'expérience suivante: Dans certains liquides oxygénés, l'iode se dissout d'abord avec une coloration violette qui vire graduellement au brun. La formation du composé brun met donc un temps mesurable. 


\section{$-626-$}

Il ne sera donc pas constitué par la ligroïne elle-même, mais par une impureté contenue dans le produit commercial. Cette impureté forme avec le premier milligramme d'iode un produit d'addition brun, mais une fois saturée elle ne réagit plus avec un excès d'iode et c'est pourquoi les solutions concentrées de cet élément ont même à basse température une couleur qui est plus près du violet que du brun.

Le produit d'addition présente le phénomène général de dissociation par élévation de température.

Pour vérifier l'exactitude de notre hypothèse, nous avons purifié la ligroïne par traitements successifs à l'acide sulfurique concentré, à la potasse caustique et au sodium métallique et nous l'avons distillée ensuite. Nous avons obtenu ainsi un dissolvant débarrassé des dernières traces de substances oxygénées et d'une façon générale de substances coordinativement non saturées. Dans ce liquide, l'iode se dissout avec une coloration d'un beau violet, qui est plus bleuâtre encore que celle que l'on obtient avec de la ligroïne ordinaire. Une solution de $1 \mathrm{mg}$. d'iode dans $10 \mathrm{~cm}^{3}$ de ligroüne purifiée présente à $-18^{\circ}$ exactement la même nuance qu'à $+30^{\circ}$.

Si nous ajoutons à cette ligroïne environ $0,2 \%$ d'alcool, nous pouvons de nouveau constater le phénomène observé par M. Dutoit. Dans cette ligroïne impure, le phénomène est toutefois moins prononcé que dans la ligroïne ordinaire. De même dans les liquides suivants, additionnés d'une trace d'alcool, le phénomène est plus ou moins visible: chloroforme, tétrachlorure de carbone, sulfure de carbone et toluène.

Lausanne, Laboratoire de Chimie organique de l'Université. 